\title{
„Zwischen Spannung und Sehnsucht" - Einblicke in forschungsgeleitete interreligiöse Lehrentwicklung unter Berücksichtigung des Aspekts Sprachsensibilität
}

\author{
Mag. ${ }^{a}$ Fatima Cavis \\ Institut für Fachdidaktik, Bereich Islamische Religionspädagogik \\ Universität Innsbruck \\ Mag. ${ }^{\text {D Dr. }}{ }^{\text {in }}$ Maria Juen \\ Institut für Praktische Theologie \\ Universität Innsbruck
}

\section{Ausgangspunkt und Kontext}

„... überall ist da irgendwo zwischen Muslimen und Christen eine Spannung und gleichzeitig auch eine Sehnsucht ..." So beschreibt eine katholische Studentin die sozio-politische Lage in Österreich. Sie hat im Rahmen des Projekts „Interreligiöse Religionspädagogik und Religionsdidaktik“ gemeinsam mit muslimischen Studierenden an einer Volksschule das Basispraktikum im katholischen Religionsunterricht absolviert. In einem qualitativen Interview, das im Anschluss an das Basispraktikum stattfand, spricht sie von einer ,großen Wunde“, von einer „aufgeheizten Stimmung“ im Land und betont gleichzeitig die hohe Bedeutung von interreligiösen Begegnungen für einen fruchtbaren Dialog zwischen Christen und Muslimen. Die Bedeutsamkeit der persönlichen Begegnung von Studierenden unterschiedlicher Religionen im Lehrveranstaltungs- und Schulkontext bestätigt sich auch in den bis dato erzielten Forschungsergebnissen des Projekts „Forschungsgeleitete interreligiöse Lehrentwicklung“, das Teil des 
umfassenderen Forschungsprojekts „Interreligiöse Religionspädagogik und Religionsdidaktik" ist.

Als ein (post)modernes Phänomen begegnet uns Vielfalt in allen Bereichen unseres alltäglichen Lebens. Die hohe Mobilität sowie die Möglichkeiten der digitalen Vernetzung begünstigen die vermehrte Begegnung und Kommunikation von Menschen aus unterschiedlichen kulturellen, sprachlichen und religiösen Kontexten in der globalen Welt. Dieses Faktum bringt neben fruchtbaren und bereichernden Erfahrungen als Ergebnis einer offenen Begegnung ${ }^{1}$ auch Herausforderungen und Probleme mit sich. Der Frage, wie dieser Vielfalt und den damit zusammenhängenden Differenzen begegnet werden kann bzw. wie damit konstruktiv umgegangen werden kann, kommt in diesem Zusammenhang eine entscheidende Bedeutung zu.

Gerade das Bildungssystem steht hier vor der Aufgabe, bei der Orientierungsfindung und beim Kompetenzerwerb im Hinblick auf den Umgang mit Pluralität mitzuwirken. Als Wissenschaftsdisziplinen, die sich mit religiöser Bildung auseinandersetzen, obliegt es auch der Religionspädagogik und Religionsdidaktik hierbei ihre eigenen Beiträge zu leisten. In diesem Sinne trägt das Forschungsprojekt „Interreligiöse Religionspädagogik und Religionsdidaktik“, das u. a. auf die Förderung grundlegender Kompetenzen im Umgang mit religiöser Pluralität und Differenz im Bildungskontext (Schule und Universität) zielt, wesentlich zur Professionalisierung von ReligionslehrerInnen in der universitären Ausbildung und in der beruflichen Fort- und Weiterbildung bei.

Das Forschungsprojekt wird vom Fachbereich Islamische Religionspädagogik am Institut für Fachdidaktik an der School of Education der Universität Innsbruck und von den Fachbereichen Katechetik/Religionspädagogik und Religionsdidaktik am Institut für Praktische Theologie der Katholisch-Theologischen

1 Ein aktuelles Beispiel hierfür ist die Fastenaktion muslimischer Jugendlicher, die in der christlichen Fastenzeit aus Solidarität mit den Christen auf bestimmte Nahrungsmittel verzichtet haben. Verfügbar unter: Link [29.03.2016]. Ebenso ist die Aussage der westlichen Feministin Miriam Gebhardt als ein bereicherndes Ergebnis einer offenen interkulturellen Begegnung zu verstehen. Sie meinte in einem Radiointerview mit Angelika Gutzeit („Sind die alten feministischen Ideale passé?“), dass muslimische Frauen mit Kopftuch den westlichen Feministinnen zur Kenntnis gebracht hätten, dass es nicht eine Form der Emanzipation gibt, sondern das Tragen eines Kopftuchs auch emanzipatorische Bestrebungen bekunden kann. Verfügbar unter: Link [29.03.2016].

Barbara Hinger (Hg.), Zweite „Tagung der Fachdidaktik“ 2015.

Sprachsensibler Sach-Fach-Unterricht - Sprachen im Sprachunterricht.

(C) 2016 innsbruck university press, ISBN 978-3-903122-51-2, DOI 10.15203/3122-51-2 
Fakultät Innsbruck unter der Leitung von Univ.-Prof. Dr. Zekirija Sejdini und assoz. Prof. ${ }^{\text {in }}$ Dr. ${ }^{\text {in }}$ Martina Kraml entwickelt. Die enge Kooperation zwischen beiden Fachbereichen begann mit der Errichtung des Studiums der Islamischen Religionspädagogik im Wintersemester 2013/14 und konkretisiert sich in Forschung und Lehre. So besuchen muslimische und katholische Studierende eine Reihe von fachdidaktischen und schulpraktischen Lehrveranstaltungen gemeinsam. Dazu zählen u. a. das Seminar „Religionsdidaktik Grundlagen“ und das anschließende „Basispraktikum“, das vier Wochen dauert und ein Praktikum an einer Volksschule sowie eine universitäre Begleitlehrveranstaltung umfasst. Ziel des Basispraktikums ist es, den Studierenden ein erstes Eintauchen in den Schulalltag und erste Unterrichtserfahrungen zu ermöglichen. Es ist Voraussetzung für das anschließende Fachpraktikum, das nicht interreligiös durchgeführt wird.

Aufgrund der Tatsache, dass es im Bereich des islamischen Religionsunterrichts bislang noch zu wenige ausgebildete Begleitlehrpersonen gibt, absolvierten muslimische und katholische Studierende im Wintersemester 2014/15 das Basispraktikum erstmals gemeinsam im katholischen Religionsunterricht. ${ }^{2}$ Die Zusammenarbeit in den interreligiös durchgeführten Lehrveranstaltungen bedeutete für Studierende und Lehrende eine bereichernde Erfahrung. Gleichzeitig stellte die muslimisch-katholische Zusammensetzung der Praxisgruppen für die katholischen BegleitlehrerInnen eine besondere Herausforderung dar, die eine hohe Sensibilität für den/die religiös Anderen verlangte.

Um eine forschungsbasierte Lehrentwicklung voranzutreiben, werden die interreligiös durchgeführten Lehrveranstaltungen derzeit qualitativ-empirisch untersucht. Die Untersuchungsergebnisse sollen für die Konzeption einer interreligiösen Religionspädagogik und Religionsdidaktik fruchtbar gemacht werden.

Der vorliegende Beitrag hat das Teilprojekt „Forschungsgeleitete interreligiöse Lehrentwicklung“ zum Gegenstand. Im Folgenden werden zunächst das Forschungsinteresse, die methodische Herangehensweise sowie die methodologische Verortung des Projekts dargestellt. An einem Fallbeispiel aus der Forschungspraxis wird in einem nächsten Schritt die Bedeutung der Sprachsensibilität für

2 In Zukunft ist geplant, dass katholische Studierende im Rahmen des Basispraktikums auch den islamischen Religionsunterricht besuchen können, sodass ein wechselseitiger Austausch möglich wird.

Barbara Hinger (Hg.), Zweite „Tagung der Fachdidaktik“ 2015.

Sprachsensibler Sach-Fach-Unterricht - Sprachen im Sprachunterricht.

(C) 2016 innsbruck university press, ISBN 978-3-903122-51-2, DOI 10.15203/3122-51-2 
eine fruchtbare interreligiöse Begegnung skizziert. Eine Zusammenfassung erster Ergebnisse schließt den Beitrag ab.

\section{Forschungsinteresse - Forschungsmethoden - Forschungsmethodologie}

\subsection{Forschungsinteresse}

Im Mittelpunkt des Forschungsinteresses steht die Rekonstruktion jener subjektiven Konzepte und intersubjektiven Dynamiken, die sich in der Interaktion und Kommunikation von Angehörigen der muslimischen und katholischen Religion in den interreligiös durchgeführten Lehrveranstaltungen und Praktika zeigen. Die empirische Untersuchung dieser subjektiven Konzepte, Muster und Dynamiken liefert grundlegende Erkenntnisse, die in die Weiterentwicklung der Konzeption der interreligiös durchgeführten Lehrveranstaltungen und Praktika Eingang finden. Diese Erkenntnisse stellen jedoch ebenso eine Fundgrube für die theoretische Grundlagenforschung dar. Dadurch können Rückschlüsse auf jene Haltungen gezogen werden, die für eine fruchtbare interreligiöse Begegnung grundlegend sind.

Die zentrale Forschungsfrage der empirischen Untersuchung lautet daher wie folgt:

- Was zeigt sich in religionspädagogischen und religionsdidaktischen Bildungsprozessen, wenn muslimische und katholische Studierende und Lehrende in Lehrveranstaltungen interagieren bzw. wenn muslimische und katholische Studierende sowie Akteure im Schulkontext (PraxislehrerInnen, SchülerInnen, DirektorInnen, Eltern) aufeinander treffen? 
Aus dieser Rahmenfrage ergeben sich folgende detailliertere Forschungsfragen:

- Welche Einflussfaktoren (subjektive Konzepte und Muster, interaktionelle Dynamiken, kontextuelle Bedingungen, inhaltliche Aspekte u. a.) können in interreligiösen Bildungsprozessen identifiziert werden?

- Welche Handlungsstrategien lassen sich bei den Akteuren erkennen?

- Welche Schlussfolgerungen ziehen die Akteure?

Mit Blick auf die Didaktik interreligiöser Bildungsprozesse stehen folgende Fragen im Fokus der Untersuchung:

- Was ergibt sich aus dem empirischen Befund für die Didaktik von religiösen Bildungsprozessen in den verschiedenen Handlungsfeldern?

- Welche hochschuldidaktischen Modifizierungen der interreligiös durchgeführten Lehrveranstaltungen und Praktika legen sich auf dem Hintergrund des empirischen Befunds nahe?

Um eine möglichst offene Herangehensweise an die Erfahrungen der Akteure im interreligiösen Interaktions- und Kommunikationsprozess zu gewährleisten, wurden die Forschungsfragen zunächst sehr allgemein formuliert. Diese offenen Fragestellungen ermöglichen ein exploratives Vorgehen, bei dem die spezifischen Forschungsfragen erst im Zuge des Forschungsprozesses entwickelt und präzisiert werden.

\subsection{Forschungsmethoden}

\subsubsection{Datenerhebung}

Zur empirischen Untersuchung der unterschiedlichen Lehrveranstaltungen wurden verschiedene Methoden der Datengewinnung gewählt, die im Folgenden in chronologischer Reihenfolge skizziert werden. 
- Legearbeiten mit Kommentar der Studierenden

In einem ersten Schritt wurde die universitäre Begleitlehrveranstaltung zum Basispraktikum evaluiert. Im Fokus stand dabei die Frage, wie die Studierenden die interreligiöse Zusammensetzung der Seminargruppe erlebt haben. Um einen mehrdimensionalen Einblick in die Erfahrungen der Studierenden zu gewinnen, wurde im methodischen Design der Datengewinnung die „Eigenproduktion visueller Daten“ (Hussy et al. 2013: 243) mit einer verbalen Befragung kombiniert. Nach Hussy et al. (2013: 243) stellt die „Eigenproduktion visueller Daten das Äquivalent [...] zur schriftlichen offenen Befragung im verbalen Bereich dar"، Forschungspraktisch wird dabei so vorgegangen, dass den TeilnehmerInnen am Forschungsprojekt möglichst große Freiheit in der Gestaltung von Bildern, Collagen oder anderen visuellen Daten (z.B. Videos) gegeben wird.

Konkret erhielten die Studierenden der universitären Begleitlehrveranstaltung in der letzten Seminareinheit von der Lehrveranstaltungsleiterin folgende Frageund Aufgabenstellung: „Wie habe ich die Lehrveranstaltung in dieser Zusammensetzung erlebt? Gestalte ein Bild und teile deine Gedanken.“ Aus einer Fülle an bereitliegenden Bildern, Gegenständen und Symbolen konnten die Studierenden ein Bild legen oder ein eigenes malen, das sie dann in der Seminargruppe kurz (ein bis zwei Minuten) kommentierten. Die Kommentare wurden digital aufgenommen und transkribiert; die Bilder fotografiert.

- Leitfadengestützte Gespräche mit Praxisgruppen

Im Anschluss an das Basispraktikum wurden drei ausgewählte Praxisgruppen in einem Gruppengespräch auf Basis eines Leitfadens zu ihren Erfahrungen in der muslimisch-katholisch zusammengesetzten Praxisgruppe, mit den BegleitlehrerInnen, SchülerInnen und anderen AkteurInnen in der Schule (Eltern, DirektorInnen) befragt. In der Zusammenstellung des Samples wurde auf möglichst große Kontrastierung geachtet. Anstelle von Einzelinterviews wurden Gespräche mit der gesamten Praxisgruppe (3 Studierende) geführt, basierend auf der Annahme, dass der Gesprächsverlauf auch Aufschluss über die Interaktionsund Kommunikationsmuster in der Praxisgruppe gibt. Von Interesse waren also

Barbara Hinger (Hg.), Zweite „Tagung der Fachdidaktik“ 2015.

Sprachsensibler Sach-Fach-Unterricht - Sprachen im Sprachunterricht.

(C) 2016 innsbruck university press, ISBN 978-3-903122-51-2, DOI 10.15203/3122-51-2 
nicht nur die explizit geäußerten Erfahrungen der Studierenden während des Praktikums, sondern auch wie sich die Interaktion in der Gruppe im Verlauf des Interviews gestaltet.

Angelehnt an die Grundprinzipien qualitativer Interviews war es ein zentrales Anliegen, den Studierenden im Gespräch ,so viel offenen Raum wie möglich zu geben, damit diese [...] ihre subjektiven Relevanzsysteme, Deutungen und Sichtweisen verbalisieren können“ (Kruse 2014: 150). Der Spannung zwischen einer möglichst großen Offenheit in der Interviewführung und einer bereits vorgegebenen Struktur durch den Leitfaden wurde dadurch begegnet, dass die Gespräche mit einer erzählgenerierenden Einstiegsfrage eröffnet wurden. Diese lautete: „Was haben Sie als Studierende in der muslimisch-katholisch zusammengesetzten Praxisgruppe in der Schule erlebt?" Ziel dieses sehr offen formulierten Erzählimpulses war es, den Interviewten „die Möglichkeit zur eigenstrukturierten Positionierung und Thematisierung“ (Kruse 2014: 217) zu geben.

Das Gespräch mit und unter den Studierenden wurde nach dem Einstiegsimpuls von den Interviewerinnen nicht moderiert, sondern die Studierenden nahmen oder gaben sich, wie im Vorgespräch vereinbart, das Rederecht innerhalb der Dreier-Gruppe. Die Interviewpersonen beschränkten sich in dieser Gesprächsphase auf die Rolle der Zuhörerinnen und brachten sich nur dort ein, wo es galt, das Gespräch in Gang zu halten. Dies geschah durch aktives Zuhören sowie durch nicht-direktive, aber das Erzählen motivierende Äußerungen wie „ja“, „mhm“ etc. (Lehner-Hartmann 2014). Darüber hinaus wurden die Studierenden ermuntert, ihre abstrakteren Aussagen durch Beispiele zu illustrieren. Erst in einem zweiten Schritt wurden - auf dem Hintergrund des Leitfadens - wesentliche, noch nicht zur Sprache gebrachte Aspekte angesprochen oder bereits erwähnte Themen aufgegriffen, um diese zu vertiefen. Die Gespräche wurden digital aufgenommen und transkribiert.

- Gruppengespräch mit Begleitlehrpersonen

Um die Haltungen und subjektiven Konzepte der BegleitlehrerInnen im Hinblick auf die interreligiöse Begegnung mit den Studierenden rekonstruieren zu können, wurde im Anschluss an das Basispraktikum auch ein Gruppengespräch 
mit den BegleitlehrerInnen geführt. Die Eingangsfrage lautete „Wie bin ich ins Basispraktikum gegangen? Was habe ich erlebt?" Die anschließende zweite Gesprächsrunde wurde mit der Frage „Wenn ich an das Basispraktikum im nächsten Studienjahr denke: Was fällt mir dazu ein?“ eröffnet. Auch dieses Gespräch wurde digital aufgenommen und transkribiert.

\subsubsection{Qualitative Datenanalyse und -interpretation}

Die gewonnenen Daten werden derzeit in einer muslimisch-katholisch zusammengesetzten ForscherInnengruppe analysiert und interpretiert (Kruse 2014). Wesentlich dabei ist, dass sowohl die muslimischen als auch die katholischen Perspektiven in den Forschungsprozess eingebracht werden und so Forschungsergebnisse in einer interreligiösen Zusammenarbeit auf Augenhöhe generiert werden. Dass dies in aktuellen Forschungsprojekten zu religiöser Pluralität und Interreligiosität im Schul- und Unterrichtskontext sowie in der LehrerInnenbildung noch keineswegs gängige Praxis ist, zeigt die Forderung des Bildungswissenschaftlers Schluß, wenn er im Hinblick auf künftige Untersuchungen meint: „Hier wären in der Weiterentwicklung der vorhandenen Instrumente (internationale) Kooperationen von Religionspädagogiken der Religionsunterrichte unterschiedlicher Konfessionen und Religionen hilfreich.“ (Schluß 2015: 92).

Forschungspraktisch wird in der Auswertung der Daten so vorgegangen, dass die transkribierten Gespräche in einem ersten Schritt einer „deskriptiven Analyse der sprachlichen Selektionen“ (Kruse 2014: 384) unterzogen werden. Im Fokus steht dabei die Beschreibung dessen, was im Text ausgesagt wird, was nicht zur Sprache kommt und wie etwas gesagt wird. Eine hilfreiche Fragestellung für diese Herangehensweise an die Texte stammt von Clifford Geertz: „What the hell is going on?" (Kruse 2014: 382). Ziel ist es, im Interpretationsprozess nicht bei der Beschreibung wahrgenommener Phänomene stehen zu bleiben, sondern die dahinterliegenden Konzepte aufzudecken (Breuer 2009). Dies geschieht, indem der Text sequenzanalytisch, also Zeile für Zeile und Passage für Passage interpretiert wird, um auf diese Weise verschiedene Lesarten zu generieren (Kruse 2014). In einem weiteren Schritt werden diese zu Kategorien verdichtet und erste Modellierungen vorgenommen.

Barbara Hinger (Hg.), Zweite „Tagung der Fachdidaktik“ 2015.

Sprachsensibler Sach-Fach-Unterricht - Sprachen im Sprachunterricht.

(C) 2016 innsbruck university press, ISBN 978-3-903122-51-2, DOI 10.15203/3122-51-2 
Die von den Studierenden in der universitären Begleitlehrveranstaltung zum Basispraktikum gestalteten Bilder werden in die Interpretation der transkribierten Kommentare einbezogen. Eine detaillierte, methodisch geleitete Bildinterpretation ist aus forschungsökonomischen Gründen und in dieser Phase des Projekts nicht möglich.

\subsection{Forschungsmethodologie}

Forschungsmethodologisch speist sich unser Vorgehen aus mehreren Quellen: Zentrales Anliegen unserer offenen Herangehensweise an den Untersuchungsgegenstand ist es, die Kategorien aus den gewonnenen Daten heraus - und damit induktiv - zu entwickeln. Dies legt die Wahl eines gegenstandsbezogenen Forschungsansatzes wie der Grounded-Theory-Methodologie nahe (Strauss \& Corbin 1996), die allerdings flexibel an die Forschungsfragen angepasst wird.

Darüber hinaus ist unsere Vorgangsweise methodologisch im Innsbrucker Planungsmodell (Scharer 2002) begründet, das auf dem theoretischen Hintergrund der Kommunikativen Theologie (Hilberath \& Scharer 2012) aufbaut und sich durch Mehrperspektivität auszeichnet. Diese Mehrperspektivität umfasst den Blick auf die Einzelnen, auf die Interaktionsprozesse in Gruppen und auf den Kontext, in dem, im vorliegenden Fall, die interreligiösen Interaktions- und Kommunikationsprozesse stattfinden. Im Forschungsprozess werden daher nicht nur die subjektiven Konzepte der Studierenden und BegleitlehrerInnen im Hinblick auf interreligiöses Handeln, sondern auch die Interaktions- und Kommunikationsprozesse im Wir der Praxisgruppe bzw. in der Zusammenarbeit der Studierenden mit SchülerInnen und BegleitlehrerInnen in den Blick genommen. Die interreligiöse Konzeption des Basispraktikums hat auch politische Reaktionen und mediale Resonanzen ausgelöst. Diese gesellschaftliche Dimension muss daher im Forschungsprozess ebenso berücksichtigt werden.

Neben der Mehrperspektivität zeichnet sich das Innsbrucker Planungsmodell bzw. die Kommunikative Theologie durch das Einbeziehen verschiedener Ebenen aus. „Forschungs- oder Lernprozesse spielen sich im Verständnis Kommunikativer Theologie auf mehreren - nicht hierarchisch zu verstehenden - Ebenen $\mathrm{ab}$, die miteinander zu vernetzen sind: auf der unmittelbaren Beteiligungsebene,

Barbara Hinger (Hg.), Zweite „Tagung der Fachdidaktik“ 2015.

Sprachsensibler Sach-Fach-Unterricht - Sprachen im Sprachunterricht.

(C) 2016 innsbruck university press, ISBN 978-3-903122-51-2, DOI 10.15203/3122-51-2 
der Erfahrungs- und Deutungsebene und auf der wissenschaftlichen Reflexionsebene" (Kraml 2015: 22). In diesem Sinne geht es in einem ersten Schritt darum, Studierende nach ihrem unmittelbarem Erleben und Erfahren der interreligiös durchgeführten Lehrveranstaltungen zu fragen und diese wissenschaftlich reflektiert und methodisch geleitet zu untersuchen. Eine zentrale Herausforderung im gesamten Forschungsprozess besteht darin, die Methodologie auf Basis der genannten Quellen interreligiös weiterzuentwickeln.

Hervorzuheben ist, dass sich das Projekt noch in der Pilotphase befindet und das Thema Sprachsensibilität nicht den zentralen Fokus der Forschung bildet. Angeregt durch die Tagung „Sprachsensibler Sach-Fach-Unterricht - Sprachen im Sprachunterricht" wurde jedoch zu Beginn des Interpretationsprozesses das Datenmaterial auch aus dieser Perspektive in den Blick genommen. Wesentliche Aspekte dieser Analyse werden im Folgenden näher dargestellt.

\section{Sprachsensibilität in der interkulturellen und interreligiösen Begegnung}

Ein Hauptthema der einschlägigen Literatur zum interkulturellen Kompetenzerwerb stellt die Frage nach den Bedingungen ,erfolgreicher' Kommunikation zwischen Menschen mit unterschiedlichem kulturellen Hintergrund dar (Erll \& Gymnich 2010; Broszinsky-Schwabe 2011; Yousefi 2014). In diesem Zusammenhang wird zur Verhinderung von Missverständnissen u. a. darauf aufmerksam gemacht, dass die Bedeutungen, die bestimmten Zeichen, Symbolen, Begriffen, Aussagen oder Verhaltensweisen zugeschrieben werden, von Kultur zur Kultur Differenzen aufweisen können. Neben dieser Kenntnis der unterschiedlichen Sinngebungen wird ferner hervorgehoben, dass Haltungen wie Offenheit, Toleranz und Empathie sowie die Erkenntnis der Kulturbedingtheit und Begrenztheit des eigenen Wissens für eine gelungene Begegnung und Kommunikation unverzichtbar sind (Erll \& Gymnich 2010; Yousefi 2014).

Dabei kann das eigene Wissen bei der interkulturellen Kommunikation insofern dialoghemmend wirken, als die DialogpartnerInnen ihr Wissen, das auf

Barbara Hinger (Hg.), Zweite „Tagung der Fachdidaktik“ 2015.

Sprachsensibler Sach-Fach-Unterricht - Sprachen im Sprachunterricht.

(C) 2016 innsbruck university press, ISBN 978-3-903122-51-2, DOI 10.15203/3122-51-2 
ihren kulturell bedingten Erfahrungen und Kenntnissen basiert, verabsolutieren. Dies kann dazu führen, dass die jeweils andere Kultur aus dem eigenen Blickwinkel heraus, ethnozentrisch bewertet wird (Yousefi 2014).

Ebenso problematisch ist die Annäherung an die fremde Kultur mit stereotypen Mustern, die zum einen historisch im kollektiven Bewusstsein der Menschen verankert sind und zum anderen häufig durch Medien in ihrer modernen Erscheinung reproduziert werden. Dieselben Probleme treten in ähnlicher Weise u. a. in der interreligiösen Begegnung auf, in der nicht die kulturelle, sondern die religiöse Identität der PartizipantInnen im Vordergrund steht.

Betrachtet man in diesem Zusammenhang den schulischen Kontext, in dem sich SchülerInnen und LehrerInnen mit unterschiedlicher kultureller Verwurzelung und religiöser Zugehörigkeit begegnen, kommt es nicht selten vor, dass SchülerInnen aus den Minderheitenkulturen in ihren Klassen von ihren LehrerInnen als die ExpertInnen ihrer eigenen Religionskultur wahrgenommen werden und dabei mit klischeehaften und stereotypen Fragen konfrontiert werden. Dies betrifft die muslimischen SchülerInnen in besonderer Weise.

Dagegen würde eine schülerInnengerechte und authentische Begegnung mit der Lebenswelt einer muslimischen Schülerin oder eines muslimischen Schülers dadurch erzielt werden, dass sie/er gebeten wird, aus der eigenen Lebenserfahrung als MuslimIn zu erzählen. Dies würde die/den SchülerIn nicht in eine schwierige Situation rücken, in der sie/er auf Fragen antworten muss, die sie/ er aus ihrer/seiner eigenen religiösen Erfahrung und Praxis in dieser Form nicht kennt. Diese Vorgehensweise ermöglicht es dem Fragenden auch zu erkennen, dass die Religionen keine monolithischen Gebilde sind und die Angehörigen der jeweiligen Religion keine homogene Gruppe darstellen.

Um sich einer fremden Kultur anzunähern oder eine andere Religion zu verstehen, ist es ergo kein angemessenes Vorgehen, wenn dies mit Fragen oder Anforderungen an die (kulturell oder religiös) Anderen erfolgt, die durch die eigenen, geschichtlich gewachsenen kulturellen Traditionen und religiösen Erfahrungen dergestalt geprägt sind, dass sie den jeweils Anderen in dieser Form unzugänglich sind. Da die fremde Kultur bzw. Religion diese Fragen aufgrund ihres Andersseins oftmals nicht kennt und daher nicht beantworten kann, könnte es zu Enttäuschungen kommen. Dagegen würde eine andere Form der Annäherung, wie

Barbara Hinger (Hg.), Zweite „Tagung der Fachdidaktik“ 2015.

Sprachsensibler Sach-Fach-Unterricht - Sprachen im Sprachunterricht.

(C) 2016 innsbruck university press, ISBN 978-3-903122-51-2, DOI 10.15203/3122-51-2 
sie von den meisten modernen IslamwissenschaftlerInnen wie Cantwell-Smith, Krawietz, Schöller und Waardenburg vorgeschlagen wird, darin bestehen, die jeweilige Kultur bzw. Religion aus ihrem Selbstverständnis heraus wahrzunehmen (Takim 2007; Schöller 2000). In diesem Sinne würde das Sich-Einlassen auf die fremde Kultur bzw. Religion oder das Sich-von-ihr-etwas-sagen-Lassen einen Zugang ermöglichen, der zu einem besseren Verständnis führt (Schöller 2000). In dieser authentischen Begegnung könnten Aspekte entdeckt werden, die als Bereicherung erfahren werden.

Im Hinblick auf das Gelingen interreligiöser Kommunikation ist es auch wichtig, sich der adäquaten Bedeutung der verwendeten Begriffe bewusst zu werden. Die hohe Bedeutung der Sprachsensibilität im Hinblick auf die verwendeten Begriffe und Inhalte lässt sich auch im Projekt „Forschungsgeleitete interreligiöse Lehrentwicklung" bestätigen. Folgendes Zitat aus der Forschungspraxis verdeutlicht diesen Sachverhalt: „Und das erste, was ich ihnen gesagt habe, war: ,Grüß Gott, ich bin die Jutta, Merve ${ }^{3}$ warum hast du kein Kopftuch an?' Weil, jetzt habe ich gesagt, jetzt haben wir so ein Tohuwabohu g'habt, wegen diesen extrem, also uns ist geschildert worden, sie sind nicht extrem, sondern sie sind einfach wirklich überzeugte Islamisten. Und jetzt kommt die ohne Kopftuch, gel.“ (Zitat aus dem Gruppengespräch mit den BegleitlehrerInnen).

Das Zitat stammt von einer katholischen Begleitlehrerin, die im Rahmen des Basispraktikums muslimische und katholische Studierende im katholischen Religionsunterricht betreut hat. In einem Nachbesprechungstreffen schildert sie vor anderen BegleitlehrerInnen und vor der Projektleitung ihre erste Begegnung mit der muslimischen Studentin. Die Lehrerin wollte sie an ihrem ersten Praktikumstag freundlicherweise vom Bahnhof abholen.

Wie das Zitat deutlich zu erkennen gibt, war die Lehrerin erstaunt darüber, dass die angehende Islamlehrerin kein Kopftuch trug. Sie konnte die muslimische Frau ohne Kopftuch mit ihrem subjektiven Bild von einer überzeugten Muslimin nicht vereinbaren. Denn nach ihrem Vorstellungsmuster musste eine überzeugte muslimische Frau ein Kopftuch tragen. Insofern impliziert die Frage der Lehrerin an die Studentin eine Aussage, die mittelbar die Glaubensüberzeugung und

3 Die Namen wurden geändert.

Barbara Hinger (Hg.), Zweite „Tagung der Fachdidaktik“ 2015.

Sprachsensibler Sach-Fach-Unterricht - Sprachen im Sprachunterricht.

(C) 2016 innsbruck university press, ISBN 978-3-903122-51-2, DOI 10.15203/3122-51-2 
somit auch die religiöse Eignung der nicht-kopftuch-tragenden, angehenden islamischen Religionslehrerin hinterfragt. Diese Begebenheit könnte die muslimische Studentin in eine Position rücken, in der sie sich für ihre Überzeugung bzw. ihre gelebte Praxis des islamischen Glaubens rechtfertigen muss.

Das Festhalten der Lehrerin an einem bestimmten Bild von einer gläubigen muslimischen Frau als Prototyp einer überzeugten Muslimin offenbart auch, dass ihr die Vielfältigkeit der muslimischen Lebenswelten nicht bekannt ist. Konkreter ausgedrückt fehlt ihr in diesem Fall die Erkenntnis, dass überzeugte muslimische Frauen die Wahl haben, sich selbstbestimmt für oder gegen das Kopftuch zu entscheiden und das Kopftuch nicht der Maßstab für den Grad der Religiosität einer Muslimin ist.

Ein weiterer, sprachlich unsensibler Punkt im Zitat der Lehrerin ist die Verwendung der Begriffe „extrem“ oder „Islamisten“ in Bezug auf die muslimischen Studierenden. Beide Begriffe sind gegenwärtig wohlbekannte Termini, die in den Medien in Zusammenhang mit den Gewaltakten islamischer Fundamentalisten verwendet werden, jedoch keinesfalls die Muslime als solche bezeichnen. Obwohl der Lehrerin dieser Unterschied aufgrund ihres Bildungsgrads bekannt sein müsste, ist in ihrer Aussage keinerlei Differenzierung auf der sprachlichen Ebene zu erkennen. Interessant ist auch, dass diese Formulierung von einer Lehrerin stammt, die dem Islam gegenüber eine offene Haltung vertritt.

Diese Beispiele zeigen, dass Sprachsensibilität im interreligiösen Kontext wesentlich ist. Sprachsensibilität in der interreligiösen Begegnung kann als Bedachtsamkeit und Sorgfalt in der Kommunikation mit dem/der religiös Anderen definiert werden. Zentral ist dabei zu beachten, was gesagt wird - also wie Inhalte und Bedeutung versprachlicht werden respektive welche Wortwahl herangezogen wird - und wie es gesagt wird.

Sprachsensible Kommunikation bedeutet auch, dass die DialogpartnerInnen auf den Interaktionszusammenhang Rücksicht nehmen sollten. Das rückt im Hinblick auf das obige Fallbeispiel die Frage in den Mittelpunkt, inwiefern die Frage der Lehrerin nach dem Kopftuch der muslimischen Studentin für eine gelungene interreligiöse Begegnung förderlich ist. Dabei ist es wenig relevant, dass die Absicht, die hinter der Frage steckt, eine gute ist. Es sollte also nicht nur die eigene Intention - die Illokution eines Sprechaktes - berücksichtigt werden, son

Barbara Hinger (Hg.), Zweite „Tagung der Fachdidaktik“ 2015.

Sprachsensibler Sach-Fach-Unterricht - Sprachen im Sprachunterricht.

(C) 2016 innsbruck university press, ISBN 978-3-903122-51-2, DOI 10.15203/3122-51-2 
dern auch die Wirkung, die die Aussage oder die Handlung bei dem/der religiös Anderen auslöst, ihre Perlokution also.

Sprachsensibilität im interreligiösen Kontext setzt voraus, dass über die Begrifflichkeiten, die verwendet werden, und deren Konnotationen Klarheit herrscht. Das ist vor allem auch erforderlich, damit, wie es das oben genannte Beispiel zeigt, keine Zuschreibungen getroffen werden, die Menschen in ,eine bestimmte Ecke rücken`. Dies erfordert wiederum neben der Empathie-Fähigkeit auch interreligiöse Kompetenz.

Die Bedeutung des (gemeinsamen) Suchens nach Begriffsklarheit zeigte sich auch in der Zusammenarbeit der Lehrenden und der Studierenden in den interreligiös konzipierten universitären Lehrveranstaltungen. Denn nicht jeder Begriff, der in der christlichen Religion verwendet wird, hat im islamischen Kontext eine idente Bedeutung. Dazu bedarf es der Bereitschaft, sich in inter- und intrareligiösen Kommunikationsprozessen der Begrifflichkeiten zu vergewissern. Sprachsensibilität auf mehreren Ebenen, angefangen von der unmittelbaren interreligiösen Begegnung über die Erfahrungsbildung bis hin zur wissenschaftlichen Reflexion, bedeutet Arbeit an und Kommunikation über die Begrifflichkeiten, die verwendet werden.

\section{Ausblick}

Erste Ergebnisse der Pilotstudie zeigen, dass sich die interreligiös durchgeführten Lehrveranstaltungen und Praktika als sehr fruchtbar erweisen. Aus den gewonnenen Daten wird deutlich, wie muslimische und katholische Studierende durch die interreligiöse Begegnung herausgefordert werden. Der anfänglichen Skepsis weicht in vielen Fällen die Erfahrung der Bereicherung durch den/die religiös Anderen. Die Möglichkeit zur authentischen Begegnung sowie die Notwendigkeit der Zusammenarbeit in den Lehrveranstaltungen und im Praktikum stoßen Identitätsbildungsprozesse an, die, wie einige Beispiele zeigen, sehr tiefgehend sind. Man kann in diesem Zusammenhang mit Schratz, Schwarz \& WestfallGreiter (2012) von einer bildenden Erfahrung sprechen, die einen wesentlichen

Barbara Hinger (Hg.), Zweite „Tagung der Fachdidaktik“ 2015.

Sprachsensibler Sach-Fach-Unterricht - Sprachen im Sprachunterricht.

(C) 2016 innsbruck university press, ISBN 978-3-903122-51-2, DOI 10.15203/3122-51-2 
Beitrag zur Entwicklung und Förderung der Pluralitätskompetenz und damit zur Professionalisierung der Studierenden leistet.

Eine wichtige Voraussetzung ist in diesem Zusammenhang, dass Lehrveranstaltungen so geleitet und gestaltet werden, dass authentische Begegnungen möglich sind. Dazu gehört, dass Differenz nicht verschleiert, sondern wertschätzend zur Sprache gebracht wird. Dies wiederum erfordert ein hohes Maß an Leitungskompetenz auf Seiten der Lehrenden, denn es gilt, nicht nur die Inhalte der Lehrveranstaltung, sondern auch die Gruppenprozesse in den Blick zu nehmen. Erste Ergebnisse zeigen darüber hinaus, wie notwendig es ist, dass auch auf Seite der Lehrenden (z.B. in der universitären Begleitlehrveranstaltung zum Basispraktikum) die muslimische und die katholische Perspektive vertreten ist, damit abwechselnd intra- und interreligiös gearbeitet werden kann.

Fort- und Weiterbildungen für BegleitlehrerInnen, in denen sie sich professionsorientiert mit dem eigenen und fremden Standpunkt auseinandersetzen können, sollten ebenso Teil einer künftigen Weiterentwicklung interreligiös konzipierter Lehre sein.

Sprachsensibilität und Arbeit an der Sprache bzw. an den verwendeten Begrifflichkeiten sind jedenfalls zentrale Aspekte im Kontext interreligiösen Lehrens und Lernens. Ihnen sollte im Rahmen der gemeinsamen Lehrveranstaltungen und Praktika noch mehr Zeit und Aufmerksamkeit gewidmet werden. Dabei gilt als Leitgedanke, was im Prinzip Grundvoraussetzung für jegliche gelungene Kommunikation ist:

„Das Interesse, der Wille und die Bereitschaft zur Verständigung mit der Absicht, den anderen in seinem Selbstverständnis zu verstehen, sind die Voraussetzungen für einen echten Dialog [...]. Das aufmerksame Zuhören, das Interesse Neues zu erfahren, ebnen den Weg einer durchgreifenden Verständigung, die eine respektvolle Haltung gegenüber dem anderen bewirkt.“ (Mohagheghi 2014: 114-115).

Barbara Hinger (Hg.), Zweite „Tagung der Fachdidaktik“ 2015.

Sprachsensibler Sach-Fach-Unterricht - Sprachen im Sprachunterricht.

(C) 2016 innsbruck university press, ISBN 978-3-903122-51-2, DOI 10.15203/3122-51-2 


\section{Literatur}

Breuer, F. (2009). Reflexive Grounded Theory. Eine Einfübrung in die Forschungspraxis. Wiesbaden: Verlag für Sozialwissenschaften.

Broszinsky-Schwabe, E. (2011). Interkulturelle Kommunikation: Missverständnisse - Verständigung. Wiesbaden: Verlag für Sozialwissenschaften.

Erll, A. \& Gymnich, M. (2010). Interkulturelle Kompetenz̧en: Erfolgreich kommunizieren zwischen den Kulturen. Stuttgart: Klett.

Hilberath, B. J. \& Scharer, M. (2012). Kommunikative Theologie: Grundlagen - Erfahrungen - Klärungen. Ostfildern: Grünewaldverlag.

Hussy, W., Schreier, M. \& Echterhoff, G. (2013). Forschungsmethoden in Psychologie und Sozialwissenschaften für Bachelor (2., überarbeitete Aufl.). Berlin, Heidelberg: Springer.

Kraml, M. (2015). Zur Methodologie des 4. Kongresses Kommunikative Theologie. In M. Juen et al. (Hrsg.), Anders gemeinsam - gemeinsam anders? In Ambivalenzen lebendig kommunizieren. Ostfildern: Grünewaldverlag.

Kruse, J. (2014). Qualitative Intervienforschung: Ein integrativer Ansat: Weinheim, Basel: Beltz Juventa.

Lehner-Hartmann, A. (2014). Religiöses Lernen: Subjektive Theorien von ReligionslehrerInnen. Stuttgart: Kohlhammer.

Mohagheghi, H. (2014). Grundlagen und Ziele einer muslimisch-christlichen Kultur der Verständigung. In H. Engin, M. Reder (Hrsg.), Wandel durch Dialog. Gesellschaftliche, politische und theologische Aspekte des Dialogs zwischen Islam und Christentum (112-118). Stuttgart: Kohlhammer.

Scharer, M. (2002). Themenzentrierte Interaktion. In G. Adam, R. Lachmann (Hrsg.), Methodisches Kompendium für den Religionsunterricht 2. Aufbaukurs (84-97). Göttingen: Vandenhoeck \& Ruprecht.

Schluß, H. (2015). Erforschung (inter-)religiöser Kompetenz. Konzepte - Probleme - neue Ideen. In H. Schluß et al. (Hrsg.), Wir sind alle ,andere“. Schule und Religion in der Pluralität (87-104). Göttingen: Vandenhoeck \& Ruprecht.

Schöller, M. (2000). Methode und Wabrheit in der Islamwissenschaft: Prolegomena. Wiesbaden: Harrassowitz Verlag.

Schratz, M., Schwarz, J. \& Westfall-Greiter, T. (2012). Lernen als bildende Erfahrung: Vignetten in der Praxisforschung. Innsbruck: Studienverlag.

Strauss, A. \& Corbin, J. (1996). Grounded Theory: Grundlagen Qualitativer Sozialforschung. (Unveränderter Nachdruck der letzten Auflage). Weinheim: Beltz. 
Takim, A. (2007). Koranexegese im 20. Jahrbundert: Islamische Tradition und neue Ansätz̧e in Süleyman Ateş's „Zeitgenössischem Korankommentar“. İstanbul: Yeni Ufuklar.

Yousefi, H. R. (2014). Grundbegriffe der interkulturellen Kommunikation. München, Konstanz: Universitätsverlag Konstanz. 
